\author{
RAMÓN PÉREZ GONZÁLEZ
}

Catedrático jubilado de la Universidad de La Laguna

\title{
Profesiones, oficios y sirvientes en La Laguna (Tenerife) en 1830
}

\section{RESUMEN}

Cerca del final del reinado de Fernando VII la población activa de La Laguna muestra los mismos caracteres que la del Antiguo Régimen. Los padrones de 1830 manifiestan el peso de los oficios; la presencia de arrieros, acorde con el arcaísmo de las comunicaciones; el número de clérigos, acrecentado por la creación de la diócesis nivariense, que supera a las profesiones liberales y a los cargos y empleos públicos. El subregistro de labradores y jornaleros impide demostrar el dominio de la actividad agraria, que es muy alto en fechas cercanas. Los criados forman una parte sustancial de la población de la ciudad, donde la segregación social apenas existe.

\section{RÉSUMÉ}

Professions, métiers et servants à La Laguna (Tenerife) en 1830.- À la fin du règne de Fernando VII la population active de La Laguna ressemble à celle de l'Ancien Régime. En 1830, le registre des habitants de la commune montre le poids des métiers; la présence des muletiers, en accord avec l'archaïsme des moyens de transport ; le nombre des prêtres, accru dès la création du diocèse nivarien, qui dépasse celui des professions libérales comme celui des postes et des emplois publics. Le sousregistre des agriculteurs et journaliers empêche attester la prédominance de l'activité agraire, pourtant très élevée à dates proches.

\section{INTRODUCCIÓN: LA FUENTE}

$E$ texto que sigue se sustenta en el análisis de los padrones de vecinos manuscritos de las dos parroquias $^{1}$ en que se dividía la ciudad, que registran los habi-

\footnotetext{
${ }^{1}$ Año de 1830. Jurisdicción de los Remedios. «Padrón del Vecindario de la Jurisdicción de $\mathrm{N}^{\text {tra }} \mathrm{S}^{\text {ra }}$ de los Remedios que se principia hoy 24 de mayo de 1830 para el Sorteo de Milicias conforme al Art $^{0} 13$ del tit. $3^{0}$ de la Real Declaración de Milicias con asistencia del $\mathrm{S}^{\text {or }}$ Correg $^{\mathrm{r}} \mathrm{y}$ de los $\mathrm{S}^{\mathrm{res}} \mathrm{V}^{\mathrm{e}} \mathrm{Ber}^{\mathrm{do}}, \mathrm{D}^{\mathrm{n}}$ Fran $^{\text {co }}$ Saviñon
}

Les servants font partie substantielle de la population de la ville, où la ségrégation sociale n'existe guère.

\section{ABSTRACT}

Professions, crafts and servants at La Laguna (Tenerife) in 1830.- At the end of the Fernando VII reign the working population of La Laguna resembles that of Ancien Régime. The 1830 register of inhabitants shows the weight of craftsmen; the presence of mule drivers, accordingly with the archaism of means of transportation; the priests number, increased with the diocese creation, which exceeds both those of white collars and public posts. The lack of registration concerning farmers and day labourers avoids to show the predominance of agriculture, nevertheless very high at close dates. Servants represent a substantial fraction of population in the town, where social segregation barely exists.

\section{Palabras Clave/Mots clé/Keywords}

Geografía urbana, siglo XIX, Canarias, padrón de habitantes, población activa, La Laguna.

Géographie urbaine, $\mathrm{XIX}^{\mathrm{ème}}$ siècle, îles Canaries, registre des habitants, population active, La Lagune.

Urban Geography, XIX $^{\text {th }}$ century, Canary Islands, register of inhabitants, working population, La Laguna.

tantes de ésta y los de su periferia más próxima, no los de todo el término municipal, realizados a lo largo de 1830. Reúnen las características propias de los recuentos preestadísticos y en lo que a profesiones se trata, el número de las registradas representa un muestra suficiente de la es-

y Guillama y $\mathrm{D}^{\mathrm{n}}$ José González de Mesa Síndico Person ${ }^{\circ}$ gen $^{1}$ [24 de mayo a 27 de octubre. Se prolonga hasta el 14 de noviembre en que se registran las últimas personas, conminadas con pena de cárcel]. Se halla concluido este Padrón de Vecind $^{\circ}$ de esta Jurisd ${ }^{\mathrm{n}}$; debiendose advertir que solo quedan sin empadronar en el 
tructura de los activos en la ciudad. A diferencia de otros muchos de la misma época, son padrones nominales por familias y casas, de manera que es posible discernir entre casas y viviendas que albergan una familia de aquellas otras que están ocupadas por dos o más núcleos familiares; especifican el grado de parentesco con el cabeza de familia, el origen de los nacidos fuera del municipio, las ausencias por emigración en número suficiente para tenerlas en cuenta en el análisis de las estructuras de la población, entre ellas los tipos de familias que la componen y los sirvientes y allegados que conviven bajo el mismo techo; también muestran las procedencias desde otros lugares de la isla de Tenerife y desde fuera de ella, y otras variables de la población incluida para muchos matrimonios la edad en que lo contrajeron; los padrones señalan asimismo el estado de las casas y los distintos usos a que se aplican; y el de las parcelas no edificadas; y en las zonas rurales si las casas ocupadas por los vecinos están en tierras de su propiedad o en suelo ajeno. En estas páginas sólo se han usado los datos que interesan a las actividades de la población, distinguiendo entre la ciudad y los núcleos, pagos, caseríos y caminos rurales cuyos habitantes se empadronan.

En lo referente a los oficios y profesiones, no se hacen constar, como queda dicho, para todos los cabezas de familia; en el ámbito urbano la información está en torno a siete de cada diez pero en los núcleos y pagos rurales la proporción desciende considerablemente; la actividad también figura para un cierto número de los descendientes lo que eleva la cantidad de registros. En una época en que el trabajo era un bien escaso cabría pensar que una parte imposible de determinar de aquellos a quienes no se asocia actividad pueden carecer de un trabajo estable con que garantizar el sustento diario para sí y su familia; y, dado el objeto de los padrones, sólo se indica su condición de paisano o miliciano, circunstancia que no ha sido estimada puesto que ser miliciano es compatible con el

valle de Tabares los Soldados Milicianos $\operatorname{Fran}^{\mathrm{co}} \operatorname{Rod}^{\mathrm{z}}$ y $\operatorname{Fran}^{\mathrm{co}}$ Migi del Rosario; en Las Mercedes Luis el Gato y Jose Agustin Guadarrama por gozar del fuero de la Guerra; y en los Genetos Tomas Suarez, Juan $\mathrm{Ant}^{\circ} \mathrm{Rod}^{\mathrm{z}}$, Cristobal Angel, Jose Antonio Adrian y Juan Angel por la propia razon de ser Milicianos, los que se resistieron a comparecer en los puntos designados $\mathrm{p}^{\mathrm{a}}$ el empadronamiento sobre los que pende expediente. Ciudad de La Laguna y Octubre 27 de mil ochocientos treinta.

Padrón del Vecindar ${ }^{\circ}$ de la Jurisdicción de $\mathrm{N} \mathrm{S}^{\text {ra }}$ de la Concepción q ${ }^{\mathrm{e}}$ se principia hoy 25 de junio de 1830 para el Sorteo de Milicias conf $\mathrm{fe}^{\mathrm{me}}$ al $\operatorname{art}^{0} 13$ del tit. $3^{0}$ de la Real Declaración de Milicias con asist ${ }^{\mathrm{a}}$ del Sr Correg ${ }^{\mathrm{r}}$ y de $\operatorname{los} \mathrm{S}^{\text {res }} \mathrm{V}^{\mathrm{e}} \mathrm{B}^{\mathrm{do}} \mathrm{D}^{\mathrm{n}}$ Antonio Padilla de Leon y $\mathrm{D}^{\mathrm{n}}$ José Gonz $^{2}$ de Mesa Síndico Person ${ }^{\circ}$ gen ${ }^{1}$. Se halla concluido este Padron del Vecindario de esta Jurisdicción, advirtiendose que solo quedo por empadronarse $\mathrm{F}^{\mathrm{co}}$ Angel de la Rosa.» Aparecen citados por Coderch (1975), que no los utiliza. ejercicio de un oficio. En el ámbito rural la proporción de quienes figuran con trabajo es mucho menor que en la ciudad y sin duda es, en gran parte, resultado de un evidente subregistro, porque la cantidad de labradores y jornaleros es tan pequeña que su número es superado por el de los que viven en la ciudad; pero también pudiera ser consecuencia de la reducción de jornaleros debida a esos años de crisis agraria.

El límite urbano de las dos parroquias separa en sentido perpendicular a las calles orientadas de este-oeste y deja a una decena de ellas perteneciendo a las dos jurisdicciones en un línea que es imposible precisar sin más referencia que los propios padrones si se desconoce el sentido de la numeración de las casas, como así ocurre; la localización de las viviendas de algunos de los personajes empadronados no es suficiente. Se prescinde, por tanto, de un análisis basado en la división eclesiástica y se realiza separando el espacio urbano del ámbito rural periférico, que se aviene mejor al análisis geográfico; aunque la calle siga constituyendo una unidad a la que recurre para la organización de gran parte de la información obtenida.

A título informativo, la cifra de habitantes censados en la parroquia de La Concepción, que es la primera y matriz de Tenerife, es de 2.716 habitantes y en la de Los Remedios, creada en 1515, después de la segunda fundación de la ciudad, es de 4.534. En el total, 7.250 habitantes, son mujeres el $56 \%$ y hombres el $44 \%$; corresponden a la ciudad 5.039 y a los pagos, caminos y lugares rurales, 2.211 .

\section{LOS GRUPOS DE ACTIVIDAD}

En los padrones aparecen 148 oficios y profesiones con las diferencias lógicas entre la ciudad y la periferia rural en lo referente al número de registros y, como se señala más arriba, a la variedad de las actividades. En lo que corresponde al espacio urbano, engloba dos plazas, siete callejones y 46 calles; salvo en las dos plazas, en cuatro callejones y once calles, todas cortas y de pocas casas y algunas, además, periféricas, en el resto hay constancia de, al menos, una profesión u oficio. En el ámbito rural, en cambio, que incluye la población de 38 lugares, pagos y caminos, en la mitad de ellos no se registra ningún activo. Este débil registro sólo se compensa, en parte, con la distinción que establecen los padrones entre quienes trabajan sus propias tierras, o al menos viven en ellas, y quienes lo hacen en ajenas; es decir, una diferencia entre propietarios y arrendadores o jornaleros de 
las tierras que trabajan para otros. Lo cierto es que ese subregistro impide conocer la magnitud de quienes viven del producto de las tierras y desvirtúa las cantidades relativas de los otros grupos. Pero, como veremos, no parece que sea éste el único conjunto de activos que no recoge a todos los que debía de haber en ellos al final del primer tercio del siglo XIX.

\section{HaCENDAdOS y TÍTULOS NOBILIARIOS}

Hay 16 hombres que se declaran hacendados. Algunos de ellos son títulos, como Fernando del Hoyo, conde de Siete Fuentes ${ }^{2}$, vinculado por matrimonio con la familia de otro hacendado, Luis F. Román, coronel del Regimiento de Milicias, que es su vecino y colindante en la calle de San Agustín. También es hacendado con título Cristóbal Salazar, conde del Valle Salazar, soltero de 40 años que vive con tres hermanas mayores, un presbítero y tres criados y que aparece como propietario de dos fincas en la periferia rural. Es título, y coetáneo de Cristóbal Salazar, Alonso de Nava Grimón (1757-1832), marqués de Villanueva del Prado; ilustrado, que tuvo jardín botánico en la calle de Anchieta, que se llamó antes de ahora precisamente «del Jardín», en referencia al mismo, y en cuyas instalaciones se reunió la Junta Suprema de Canarias en 1808; fue constante defensor de los derechos históricos de La Laguna. Otros hacendados son Lorenzo Montemayor; José Vanderhede; Baltasar Peraza, que emparenta por casamiento con la familia del Hoyo; Jorge Fuentes, de 33 años, soltero, el más joven del grupo, mozo de casa abierta que vive con una criada de 14; un Francisco Antonio; Juan Colombo Riquel y Juan Nepomuceno Machado, capitanes de Milicias; Alejandro Saviñón; Juan García y José González de Mesa, síndico personero; Mateo Fonseca y José Manuel de Castilla, nacido en América y casado allí.

Otros títulos que no aparecen empadronados pero sí como propietarios de tierras son el marqués de San Andrés, nacido en La Laguna en 1778, con casa en la calle del Agua, y el marqués de Casa Hermosa, con dos fincas cada uno, y el marqués de la Candía, con tres. Los mar-

\footnotetext{
${ }^{2}$ El condado de Siete Fuentes fue concedido en 1868 por Carlos II. El onceavo conde, Felipe del Hoyo Machado Solórzano (1920-2008), es, en el siglo pasado, un claro exponente de la intervención de este grupo social en el campo de la gestión política y de las actividades más rentables de la economía insular. Fue alcalde de Puerto de la Cruz, fundador del Centro de Iniciativas y Turismo y presidente de Ashotel, que representa y defiende los intereses de más de doscientos establecimientos hoteleros y extrahoteleros de la provincia de Santa Cruz de Tenerife.
}

quesados de Casa Hermosa y de la Candía recaen más tarde en las familias Cologan y Machado, de origen irlandés la primera, descendiente de Bernardo Walsh (Valois) Carew, exportador de vino y comerciante con Europa y América que se establece en Tenerife en 1684; y de origen portugués la segunda, cuyo ascendiente se asienta ya en la isla en fecha tan temprana como 1498. Son ejemplo de cómo a través de mayorazgos y de alianzas matrimoniales se vinculan entre sí las élites locales. Son también propietarios en las afueras de La Laguna la marquesa de San Andrés, con casa en ciudad, y el marqués de Villafuerte.

Todos los hacendados residen en calles céntricas de la ciudad: cuatro en la Carrera y en San Agustín, dos en Álamos y en Agua y uno en cada una de las calles de Herradores, Moya, Santo Domingo y plaza del Adelantado, en la que hay además de la de Juan Manuel de Castilla otras dos casas habitadas: una por el médico racionero de la catedral, tres hermanas, un sobrino estudiante y dos criadas, y la otra por una viuda joven y sus tres hijos, en cuyos bajos hay granero.

\section{LA CREACIÓN DE LA DIÓCESIS NIVARIENSE Y EL CLERO}

El Obispado de La Laguna se crea el 21 de diciembre de 1819 (Quintana, 2009, p. 243), no sin oposición del Cabildo de Gran Canaria y del Obispado de Canarias, cuya diócesis abarcaba todo el archipiélago; entre otras razones, por la cuestión de las rentas de la diócesis, la mitad de las cuales procedía de Tenerife y algo más de otra décima parte de las tres islas occidentales (Quintana, 2009 , p. 247). Como la mayor parte de la población y de las iglesias estaban en Tenerife, la isla tenía un gran peso en el total de las rentas percibidas por el Obispado; éste fue suficiente motivo para que, antes de la creación de la diócesis de La Laguna, algunos obispos de Canaria residiesen en la isla en el siglo XVIII durante largos periodos, incluso la mayor parte del tiempo de su prelatura, y dos de ellos fallecieron en la isla; de modo que la presencia de los obispos de Canaria en Tenerife fue vista con naturalidad (Cioranescu, 1977, I, pp. 177-178).

A la cabeza de la nueva diócesis se suceden tres vicarios capitulares hasta el nombramiento del primer obispo, Luis Folgueras y Sion, asturiano de Pravia (1769-1859), deán de Ourense desde 1805, que ocupó la sede entre 1825 hasta 1844 (Núñez, 1978, p. 136). Corresponden a la diócesis las islas de Tenerife, La Palma, La Gomera y El Hierro, y el Cabildo catedral debía tener seis dignidades, un deán, tres arcedianos (de Tenerife, La Palma y La Gomera), un chantre, un tesorero, catorce canónigos 


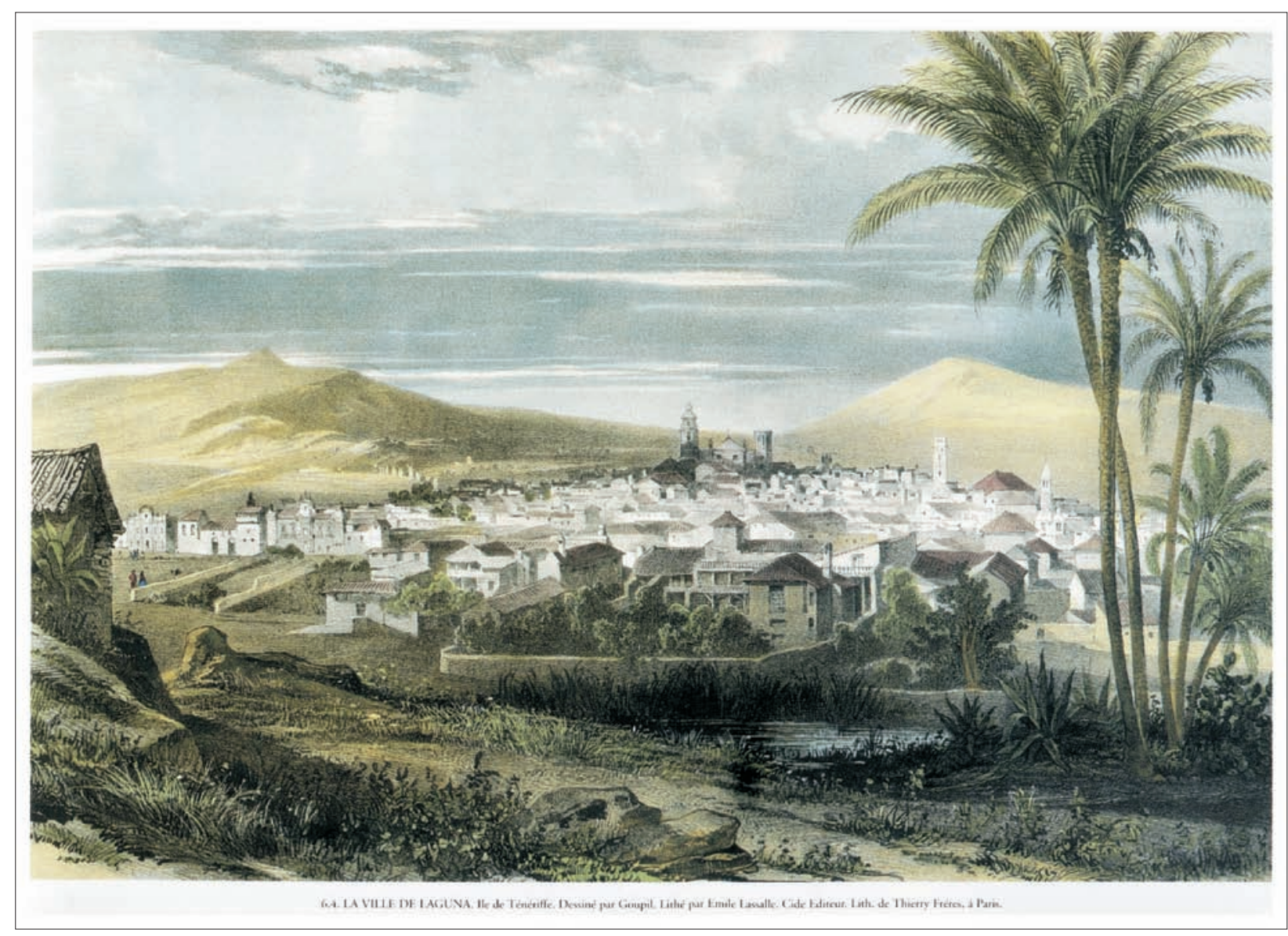

FIg. 1. La Ville de La Laguna. Île de Ténériffe. Dessiné par Goupil. Lithé par Emile Lassalle. Cide Editeur. Lth. de Thierry Frères à Paris. Litografía perteneciente a la obra Voyage au Pôle Sud et dans l'Océanie, 1847. Las obras de la torre norte de la catedral se detuvieron en 1835 y Goupil murió en 1841, de modo que el dibujo corresponde a una fecha entre esos dos años. Vista hacia el noroeste, destacan en la ciudad, en primer plano, la casa y huerta del marqués de San Andrés, del siglo XviII. En segundo plano, a la izquierda, las fachadas del ayuntamiento y el convento de las catalinas, frente a la plaza del Adelantado y de la casa de Alonso de Nava y Grimón. Más a poniente sobresalen las torres de la catedral, de la Concepción y del convento agustino. La línea de horizonte, que es muy baja y no permite seguir el trazado de las calles, se cierra con el Llano de La Laguna y a su izquierda la cordillera dorsal de la isla, sobre la que asoma el pico de Teide (Tous Meliá, 1996).

más cuatro de oficio: penitenciaría, doctoral, magistral y lectoral, diez raciones enteras y ocho raciones medias (Núñez, 1978, p. 126). El obispado se suspende después en 1851, por la firma del Concordato de ese año, hasta 1877 , en que se restablece de nuevo.

Al primer obispo, cuya prelatura se confirmó en 1824 y se prolongó hasta 1848 , le correspondió vivir la reducción en la recaudación de los diezmos, los conflictos entre grupos oligárquicos de la isla, la laicización social y la desamortización de 1836 en un escenario de constante oposición del coro catedral a su gestión (Quintana, 2009, p. 249).

La sede episcopal de los primeros años ocupó la casa 28 de la calle de la Carrera y después de restablecida la diócesis, en la calle de Bencomo, que es en 1830 la de Moya, y luego en el edificio del convento agustino. La actual sede de la calle de San Agustín fue casa de los condes de Valle Salazar y residencia del capitán general de Canarias. La adquirió para su sede el obispo Ramón Torrijos Gómez a finales del siglo XIx (Cioranescu, 1965, p. 163).

La residencia del obispo en La Laguna y la conversión de la iglesia de los Remedios en catedral debería haber supuesto un aumento del número de clérigos, de sirvientes de éstos y de trabajo para artesanos y oficios vinculados con la iglesia y la liturgia, además de lo que pudieron representar para la economía urbana las rentas catedralicias. El número de eclesiásticos y servicios y 


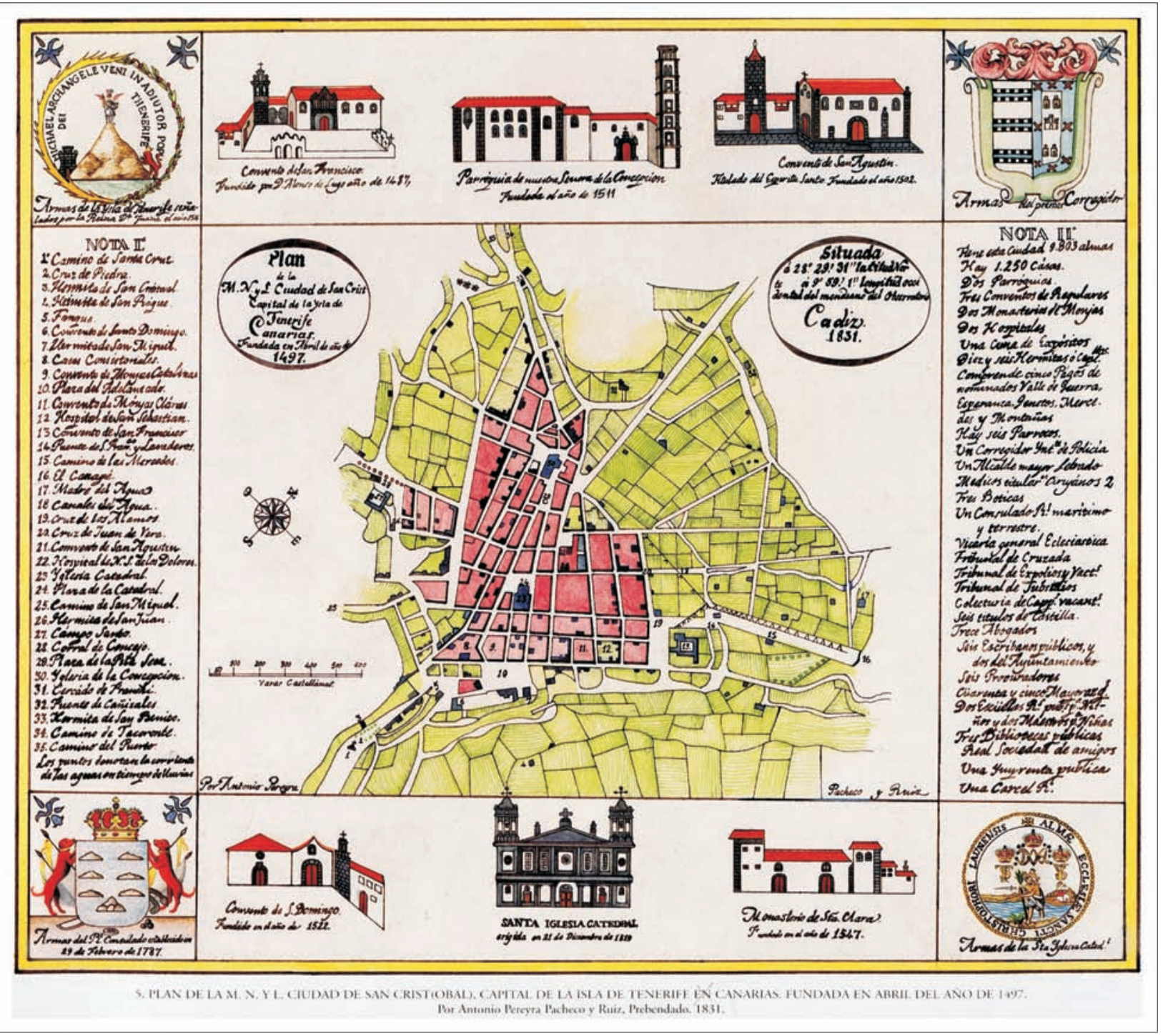

Fig. 2. Plan de la M. N. y L. Ciudad de San Crist(obal), Capital de la Isla de Tenerife. Canarias. Fundada en abril del año 1497. Por Antonio Pereyra Pacheco y Ruiz, Prebendado, 1831.

1. Camino de Santa Cruz

2. Cruz de Piedra

3. Hermita de San Cristóbal

4. Hermita de San Roque

5. Tanque

6. Convento de Santo Domingo

7. Hermita de San Miguel

8. Casas consistoriales

9. Convento de monjas catalinas

10. Plaza del Adelantado

11. Convento de monjas claras

12. Hospital de San Sebastian

13. Convento de San Francisco
14. Puente de San Francisco y Lavaderos

15. Camino de las Mercedes

16. El Canapé

17. Madre del Agua

18. Canales de Agua

19. Cruz de los Álamos

20. Cruz de Juan de Vera

21. Convento de San Agustín

22. Hospital de Nuestra Señora de los Dolores

23. Iglesia catedral

24. Plaza de la catedral

25. Camino de San Miguel

26. Hermita de San Juan
27. Campo Santo

28. Corral del Concejo

29. Plaza de la Pila Seca

30. Iglesia de la Concepción

31. Cercado de Franchi

32. Fuente de Cañizares

33. Hermita de San Benito

34. Camino de Tacoronte

35. Camino del Puerto (de la Orotava)

Los puntos denotan la corriente de las aguas en tiempo de lluvias 
oficios vinculados que incluyen los padrones son los que siguen. En la sede episcopal, además del titular, viven un presbítero y secretario, un canónigo tesorero, dos capellanes, un paje de menores y cinco criados. Además del obispo y de quienes viven con él, entre clérigos y empleados relacionados con la iglesia se cuenta 75 personas. Dos arcedianos, de la catedral y de La Palma, cuatro canónigos y cuatro beneficiados, 24 presbíteros, que incluyen al fiscal y al provisor del obispo y a otro de la catedral. En ésta hay también un capellán y un racionero. Viven en la ciudad otros dos prebendados, uno de ellos el electo de Canarias y Antonio Pereira Pacheco, autor del plano que ilustra estas páginas; dos curas del Sagrario y cuatro capellanes, uno de la catedral. Siguen un diácono, seis subdiáconos, fraile y tonsurado con beneficio eclesiástico. Además, cuatro sochantres, uno soltero de veinte años que es también sacristán, y tres casados con familia, tres músicos de la catedral, tres contadores, uno de ellos diputado del Común, escribiente del cabildo, dos campaneros y monaguillo.

No había seminario, que estaba en Las Palmas. De los conventos apenas nada; en el de Santo Domingo se empadronan el cocinero, un fraile, un prebendado, un bachiller que ha estudiado en Madrid y un músico de la catedral y, en el Santa Catalina de Siena, sólo el mandado de las monjas, que es miliciano retirado y vive con su mujer y tres hijos. Como referencia lejana, a principios del siglo XIX había en la ciudad 18 frailes profesos, un novicio y ocho legos en el convento franciscano, y 20 agustinos y 27 religiosas, dos novicias, dos legas y una donada, en el convento clariso; entonces también sin datos de los conventos dominicos de Santo Domingo y Santa Catalina (Hernández, 1983, vol. 3, p. 103).

\section{LOS CAMPOS PROFESIONALES: ENSEÑANZA, MEDICINA Y LEYES}

En 1791 las respuestas de los ayuntamientos al corregidor de la isla para un informe solicitado por el Consejo de Castilla sobre el estado de la enseñanza pública muestran una lamentable realidad de la educación primaria, que por razones fácilmente entendibles es suavizada en las contestaciones que en común remiten los alcaldes y curas de los pueblos. De otra parte, la cifra de 34 escuelas y maestros, haciendo cuenta de las dotadas y servidas por conventos, curas, sochantres, sacristanes y algún familiar de éstos, más amigas, y aun contando las casas de preceptores, parece estar por encima de la realidad. De todas éstas, las dotadas con fondos públicos, y son sólo de niños, las hay en Icod, La Orotava, Santa Úrsula; y en La Laguna, que a finales del setecientos tenía dos de niños dotadas y cuatro de niñas atendidas por mujeres de Ayuda Sociedad Patriótica (Santos y Vega, 2006a, pp. 666-667 у 675).

Después de 1814 y la condena al proceso constituyente se derogó el Reglamento General de Instrucción Pública de 1821 para devolver a la Iglesia el control de la enseñanza pública (Santos y Vega, 2006b, p. 221). Antes, en 1815 se había formado una junta a la que se encargó un nuevo Plan de Escuelas de Primeras Letras, que señalaba en La Laguna una sola escuela de esta clase, que estuvo instalada desde finales del XVIII en la casa de los jesuitas (Calero, 2001, p 55) y la dotada por oposición y regentada entonces por un maestro interino que se mantuvo hasta su muerte en 1847. Al maestro le asistía un ayudante y recibían 200 pesos y 18 fanegadas de trigos y 80 pesos y 9 fanegadas, respectivamente. Como en tantos otros núcleos urbanos, La Laguna contó con escuelas privadas en las que la enseñanza se ejercía con frecuencia por particulares sin conocimiento de los más elementales principios didácticos y pedagógicos y en locales reducidos sin condiciones de higiene y mal ventiladas, como se denuncia en Santa Cruz (Cioranescu, 1977, I, pp. 103104). Los padrones de 1830 sólo recogen a una maestra de niñas, dos maestros de dibujo y uno de primeras letras, y dotada de niños se ocupó por un interino hasta casi la mitad del siglo xix. En cambio, un año más tarde, en el plano de Pereyra Pacheco y Ruiz, de 1831, en la parte derecha de la orla, en la nota II, se lee «dos Escuelas R[eale] s pub[lica]s p[ar]a Niños y dos Maestras p[ar]a Niñas» ${ }^{3}$. Exigua dotación escolar para una población infantil que entre ciudad y periferia rural alcanzaba los 1.633 habitantes de cinco a catorce años (831 varones y 802 mujeres).

Pero la Junta Inspectora de Escuelas da cuenta de diez escuelas, tres de niños y niñas, una de niñas y cuatro de niños en 1828 y las mismas en 1830, aunque en dos no figuran alumnos. En dos escuelas de niños al maestro los nombraba el Ayuntamiento y el resto eran particulares, dos regentadas por hombres a las que sólo asistían niños y seis por mujeres; todos sin título. El número de escolares era de 204 niños y 74 niñas y en 1830, 245 y 120 , respectivamente (Santos y Vega, 2006b, p. 223). Sin duda, los padrones registran a menos maestros de los que ejercen en la ciudad. En los medios rurales la asistencia

\footnotetext{
3 «Plan de la M. N. y L. Ciudad de S. Crist[obal] Capital de la Ysla de Tenerife Canarias. Fundada en Abril de año de 1497 por Antonio Pereyra Pacheco y Ruiz, Prebendado, 1831.» Véase Tous Meliá (1996)
} 
CuADro I. Distribución de las profesiones y oficios en diez calles centrales de la ciudad según los padrones

\begin{tabular}{|c|c|c|c|c|c|c|c|c|c|c|c|c|c|c|}
\hline \multirow[b]{2}{*}{ Profesiones y oficios } & \multicolumn{11}{|c|}{ Calles } & \multirow[b]{2}{*}{$\sum_{\infty}^{\varangle}$} & \multirow[b]{2}{*}{ 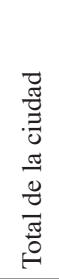 } & \multirow[b]{2}{*}{ 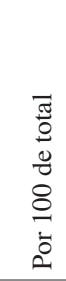 } \\
\hline & $\begin{array}{l}\tilde{O} \\
\stackrel{\Xi}{\Xi} \\
\text { \& }\end{array}$ & 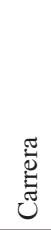 & 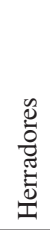 & 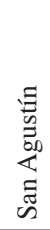 & $\stackrel{\pi}{\stackrel{\pi}{\Sigma}}$ & 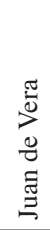 & હ & 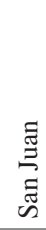 & $\underset{\overparen{Z}}{\mathbb{2}}$ & త్ర & 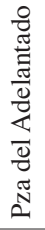 & & & \\
\hline Oficios & 45 & 12 & 37 & 11 & 16 & 13 & 6 & 6 & 8 & 1 & & 155 & 231 & 67,1 \\
\hline Comercio & 14 & 7 & 28 & 3 & 5 & 1 & & 5 & 1 & 4 & & 68 & 91 & 74,7 \\
\hline Clero & 1 & 17 & 8 & 9 & 9 & 6 & 7 & & 3 & 1 & 1 & 62 & 73 & 84,9 \\
\hline Ejército & 1 & 13 & 3 & 9 & 2 & 2 & 1 & 2 & 1 & & & 34 & 36 & 94,4 \\
\hline Leyes & 1 & 7 & 3 & 7 & 6 & & 3 & 2 & & & & 29 & 37 & 78,4 \\
\hline Jornaleros & & 1 & 3 & 1 & 2 & & 5 & 3 & 5 & 4 & & 24 & 76 & 31,6 \\
\hline Labradores & 3 & & 13 & & & 1 & 3 & 2 & & & & 22 & 29 & 75,9 \\
\hline Hacendados & 2 & 4 & 1 & 4 & 1 & & & & 2 & 1 & 1 & 16 & 16 & 100,0 \\
\hline Cargos públicos & & 3 & 1 & & 3 & 1 & & 3 & 1 & & & 12 & 16 & 75,0 \\
\hline Medicina & & 2 & & 4 & 1 & 3 & 1 & & & & & 11 & 11 & 100,0 \\
\hline Enseñanza & 1 & 1 & & & & 1 & & & & & & 3 & 4 & 75,0 \\
\hline Subalternos & 1 & & 1 & & & & & & & 1 & & 3 & 11 & 27,3 \\
\hline Suma & 69 & 67 & 98 & 48 & 45 & 28 & 26 & 23 & 21 & 12 & 2 & 437 & 631 & 69,3 \\
\hline
\end{tabular}

era poca porque los niños «están ejercitados en guardar los ganados, en sus sementeras y cosechas de granos y papas», y en La Laguna, como ocurría en Santa Cruz, «por no tener sus padres aún para el preciso alimento, les es imposible subvenir a pagar maestro; y no habiendo entretenimiento para niños de corta edad, como sucede en los pueblos de labranza y cría de ganados, andan por las calles aplicándose a los vicios que mejor les acomoda en su pequeño estado» (Santos y Vega, 2006a, p. 680).

La ciudad contó, desde finales del siglo XVIII, con universidad. Una agustina que sólo funcionó entre 1744 y 1747, a la que sucedió la Literaria de San Fernando, que, creada en 1792, abrió sus puertas en 1817 con estudios de filosofía, teología y derecho en el antiguo colegio jesuita y desde 1821 en el convento de San Agustín. Restaurado el régimen absolutista, dejó de funcionar entre 1823 y 1825 , año en que se volvió a abrir y cerrar, volviendo a funcionar en 1834. Otra vez se suprime en 1845 y al año siguiente se crea el Instituto de Segunda Enseñanza de Canarias, del que dependieron hasta la primera década del siglo $\mathrm{xx}$ todos los centros privados de las islas. Tan efímera, poco afortunada, precaria e inestable universidad no deja huella entre las profesiones de la ciudad. Tan sólo una decena de estudiantes: cuatro de la universidad y el resto simplemente estudiantes; ningún profesor empadronado pero sí un bedel y un portero de la universidad entre los subalternos. En el informe que se solicita en 1830 el corregidor responde que en relación con los alumnos que tienen las cátedras de latinidad se hallan en la universidad sujetos al rector (Santos y Vega, 2006b, p. 223 , n. 6). Entre 1825 y 1830 la matrícula no pasó de 62 alumnos y había 52 el segundo de esos años (Pérez 1971, apéndice II).

La medicina, como la enseñanza, tampoco da muchos empleos. Los médicos son ocho, de los que cinco son racioneros, tres de ellos de la catedral; los tres restantes son uno titular y otro titular interino, madeirense que vive con su prima compartiendo casa con una familia y habrá que suponer de ambos que son de nombramiento del Cabildo; del tercero nada se dice. Además, hay dos licenciados en farmacia, uno de ellos mozo de casa abierta, sin saber si tiene establecimiento, y un boticario. Todos los de profesionales de este grupo se concentran en sólo cinco calles del centro de la ciudad.

Al residir el Cabildo en La Laguna, los escribanos de la ciudad lo eran de toda la isla, pero algunos de ellos prefirieron, desde mediados del siglo XVIII, residir en Santa Cruz. Sin embargo, hay periodos en que no los hay en el 
puerto, lo que se puede explicar por la cercanía de La Laguna, donde es frecuente que los documentos notariales correspondan a personas que residen en Santa Cruz (Cioranescu, 1977, I, p. 163). Los agrupados en el epígrafe de leyes son 37 repartidos en tres notarios, dos públicos y uno judicial; un licenciado en leyes en Madrid, un bachiller, también notario público, y un bachiller en leyes; cinco abogados y cinco letrados; cuatro procuradores; ocho escribanos, de los que dos son del Cabildo, y nueve escribientes.

\section{EJÉRCITO REAL Y MILICIAS}

Mediado el siglo XVIII la guarnición de Tenerife la formaban las milicias provinciales; la presencia del Ejército real no era permanente y reforzaba a las milicias en casos de peligro de guerra. Así, después del ataque de Nelson en 1787, por ejemplo, o para garantizar el orden público. Con las tropas regulares, las milicias aseguraban la defensa y tenían al batallón de Canarias de columna vertebral. En dos ocasiones en el siglo XIX se enviaron a Tenerife tropas de los ejércitos reales: la primera vez en 1789 en que se envían dos regimientos (América y Ultonia) para hacer frente a posibles ataques ingleses; el primero volvió a la Península en abril de 1802 y el segundo en agosto de ese mismo año, quedando en la isla dos banderas (de La Habana y Cuba). En marzo de 1827 desembarca, esta segunda vez para preservar el orden público ante una posible rebelión popular, el 7. ${ }^{\circ}$ Regimiento de Infantería Ligera, Albuera. La rebelión no se produjo entre la población sino en el propio regimiento, que hubo de depurar a los sediciosos, lo que duró tres años ${ }^{4}$. En 1830 uno de los batallones se envió a La Habana y quedaron dos compañías, una en La Palma y otra en Tenerife (Cioranescu, 1977, Iv, pp. 62-64).

Así pues, las milicias canarias fueron cuerpos de reserva de la infantería cuya existencia en España se prolonga hasta su disolución en 1867. Pero el carácter de frontera del archipiélago y el peligro que suponían los ataques de piratas y las guerras hicieron que sus unidades fueran en las islas más de primera línea en la defensa del archipiélago. Eran autónomas porque los cabildos debieron organizarlas en cada isla en coherencia con los peligros a que estaba expuesta cada una de ellas. Su mando correspondía a un cuerpo eventual de oficiales que eran

\footnotetext{
${ }^{4}$ En uno de los padrones se registra a un desertor del Regimiento de la Albuera.
}

elegidos. Los capitanes, a diferencia de la Península, en que lo eran por nombramiento real, correspondían a los cabildos insulares, que lo hacían entre personas de la hidalguía y las clases acomodadas de limpia calidad, práctica y experiencia en el ejercicio de las armas; extremo este último que no era siempre el caso. Los capitanes nombraban a los alféreces, sargentos y cabos de su compañía. El alistamiento era universal y tenían la obligación de servir en filas todos los hombres de 16 a 60 años.

A principios del siglo XIX, además del Batallón de Infantería de Canarias, del Ejército regular, creado en 1799 con sede en Santa Cruz, había en la isla cinco regimientos de infantería de las milicias (La Laguna, La Orotava, Garachico, Güimar y Abona) y seis compañías de artillería. Entonces el reclutamiento se había reducido a los hombres entre 18 y 40 años y el número de milicianos en el archipiélago pasaría de unos 11.500 en 1771 a 8.400 en 1844 y sólo se contaba un miliciano por cada seis familias. Las milicias provinciales desaparecieron en la Península en 1867 y en Canarias en 1886, donde se sustituyeron por seis batallones de reserva (Abad, 2013, passim) y sus oficiales pasaron a la escala activa de infantería y al ejército territorial de Canarias (Cioranescu, 1977, IV, p 67). Los padrones registran 32 militares desde tres coroneles a un pífano; uno de los coroneles es del Rey y otro el coronel del Regimiento de Milicias de Güimar, a un pífano. Hay un teniente coronel y 19 capitanes, uno del Ejército y el resto de las milicias, como los demás oficiales; tres cadetes, uno de milicias y antes del pífano un tambor, también de milicias.

\section{CARgos PÚblicos y SUbalternos}

El número de cargos públicos y de empleados es muy reducido: el corregidor, José Berriz de Guzmán, vinculado por casamiento con la familia Román, en cuya casa se empadronan el matrimonio, siete hijos, los dos mayores guardias de S. M. y los dos que siguen cadetes de caballería y cuatro criados; el alcalde mayor y el alcaide de la cárcel; los administradores de la Alhóndiga, de la Estafeta, el administrador general de Rentas decimales de la provincia; dos diputados del Común, uno de ellos labrador y propietario de seis yuntas; un regidor perpetuo, un cargo honorífico de la Real Audiencia; dos alcaldes de barrio, uno de ellos herrero, y tres alguaciles reales.

El trabajo de los subalternos está asociado a un nivel bajo y son únicamente diez. Fiel de carnicerías, bedel y portero de la universidad, dos porteros del Cabildo y uno del Consistorio; un guarda de montes y es probable que 
con nombramiento del cabildo, un montaraz y un apeador; y un pregonero. Ninguno vive en el núcleo central de la ciudad.

\section{LOS OFICIOS Y EL COMERCIO}

La lista de oficios incluye a una cincuentena, pero en la quinta parte sólo aparece ejerciéndolo una persona; bien porque produce para una demanda reducida (chocolatero, impresor, dibujante...), bien porque se trata de oficios insanos (limpiador de cloacas, sepulturero, que es negro) y pude bastar un hombre para desempeñarlos; en otros casos, como en el numeroso grupo de la construcción, no es fácil explicar que haya un único albañil, un solo encalador, o un único tejero, aunque pueda ser ésta una actividad itinerante, extremo del que no se tiene constancia. Sí, en cambio, que al pedirse explicaciones al Ayuntamiento por el reparto de terrenos en la laguna propiamente dicha, se recuerda que no habría sitio para el pasto de animales ni otro lugar de donde sacar la tierra para teja y ladrillo (Sánchez-Manzano, 2007, p. 34). Aún otro grupo de oficios no supera tres empadronados (barberos, latoneros, cocineros, fragüeros, toneleros..., por ejemplo); son cuatro los albarderos, que hay que asociar a la arriería y a las venta a gentes de fuera de la ciudad; los plateros, único oficio de bienes de lujo, son cinco, uno de ellos con lonja, y cuyo trabajo guardará relación con la demanda de las iglesias; siete sombrereros; pero ningún sastre en los padrones, en contraste con los 42 zapateros, uno francés, y dos aprendices: la localización de sus talleres, con ocho de cada diez en el área central de la ciudad, marca una evidente concentración que hace pensar en la demanda que generan las clases altas; y 12 herreros, cinco de ellos en la calle de Herradores, que con dos latoneros forman el grupo de oficios del metal.

Al unir los oficios por grupos de actividad la construcción agrupa a 40 activos, con predominio de pedreros, que son 33, más tres canteros y dos cabuqueros, oficio que guarda relación con la construcción de pozos pero también de aljibes, de los que contaban numerosas casas de la ciudad; además, el tejero, el encalador y el albañil ya reseñados. Asociados a la construcción, pero no sólo a ella, La Laguna cuenta con 41 carpinteros, entre los que se encuentra el portero del Cabildo, y un aprendiz, que son requeridos por el amplio uso de la madera en las casas, bien sean de una o, en especial, de dos y tres plantas, tanto para obra nueva como para el mantenimiento de tillados de los pisos, las vigas, tirantes y entablazón de las cubiertas, vigas carreras para las escaleras, los balcones a la calle, la galerías en torno a los patios y los artesonados de las viviendas de las clases acomodadas y de las iglesias. Aparte los muebles, y ciertos aperos agrícolas.

Es también numeroso el de los oficios relacionados con el transporte, basado en la arriería, que debe usar una red de comunicaciones arcaica. En esta rama aparecen dos carreteros, siete conductores; doce camelleros y trece arrieros que forman más del $70 \%$ de los 34 censados; en la zona central sólo aparecen tres en Herradores y uno en Álamos.

En el comercio el establecimiento más extendido es la venta; eran 50 y expondrían a los clientes un conjunto de productos numeroso y heterogéneo de entre los que habrían de destacar los alimentarios, incluido el pescado salado, porque de las nueve pescaderías de la ciudad sólo dos lo venden en salazón y era de consumo generalizado. De otra parte, la del pescado es un caso evidente de concentración espacial de venta específica: en la calle de Chávez, que abarca sólo dos manzanas, hay seis.

Las ventas tienen presencia en casi todos los rincones de la ciudad, lo que no impide que en la calle de Álamos y de Herradores esté la mitad de ellas. La primera es prolongación del camino de las Mercedes, que viene de las tierras septentrionales de la Vega y continúa, al atravesar la ciudad, por el camino que va a Geneto y Los Baldíos; la segunda es, con su prolongación la Empedrada, que en el padrón de la Concepción aparecen unidas, la calle que encauza el tráfico del norte de la isla hacia el puerto de Santa Cruz. En Álamos diez de los catorce establecimientos son ventas y llegan a la mitad de los 22 establecimientos en Herradores, donde están también cuatro de las ocho lonjas de la ciudad, una de ellas la del platero.

Las tiendas son siete, cuatro en Carrera y tres en $\mathrm{He}$ rradores, y habrán representado un cierto grado de especialización y, por tanto, un nivel mayor que el de las ventas. En un pequeño número de estos dos tipos de establecimientos aparece la mujer: en Herradores hay tres venteras y dos tenderas y en San Antonio una de las dos ventas también está en manos femeninas. Aparecen sólo dos comercios y están en Herradores. Se pueden asimilar a este grupo siete tabernas y otros tantos molinos, donde el vino, la harina y el gofio son objeto de venta al por menor. Algunos de estos establecimientos está en manos de franceses: el tonelero, el fondero y el dueño de una de las lonjas de la Carrera son franceses, así como el molinero de la calle de San Juan. Junto al médico madeirense y su familiar son los únicos extranjeros empadronados. Menos que en el pasado; al finalizar el siglo XVIII, había en la isla un centenar y once correspondían a La Laguna, algunos de los cuales eran comerciantes pero de produc- 
tos orientados a mercados exteriores, que en el pasado nunca estuvieron en manos canarias (Cioranescu, 1977, I, p. 108).

\section{LABRADORES Y JORNALEROS: LA EXTENSIÓN DE LAS TIERRAS CULTIVADAS}

La presión demográfica, la indigencia de la población campesina y la coyuntura alcista de los precios agrícolas están en el origen de los repartimientos de tierras que se producen en La Laguna a partir de 1780. Unas veces son tierras concedidas por el rey, como es el caso del marqués de Casahermosa o del marqués de Villafuerte; éste, vecino de Garachico, recibe 300 fanegadas; o las 150 que recibe el subteniente de milicias Pedro Valdés, ambos en el Llano de San Juan. Otras son otorgadas por el Cabildo, que reparte tierras a 80 labradores, 100 jornaleros, 70 artesanos y 132 pudientes. Estos repartimientos, tomados de las dehesas y de los propios de Los Baldíos, Llano de San Juan, Llano de San Benito, Llano de La Laguna y trasera del convento de San Francisco, hacen aumentar las tierras de cultivos en casi mil fanegadas; es decir, unas 500 ha entre 1800 y 1920. Las proporción de tierras cultivadas del municipio era mayor que la media de la isla, teniendo en cuenta que hasta 1821 el municipio de El Rosario perteneció al de La Laguna (Sánchez-Manzano, 2007, pp. 33-34).

La agricultura era la actividad productiva sobre la que se sostenía la economía lagunera y en el trabajo y tenencia de la tierra intervenían la burguesía agraria, pequeños propietarios, medianeros y renteros y, sobre todo, jornaleros, que en el mejor de los casos pudieron alternar su trabajo entre los cultivos del sector exportador y el de abastecimiento del mercado interior. Las tierras que rodean La Laguna se dedicaban en más de la mitad al trigo, una cuarta parte a las papas y otro tanto al viñedo. El cultivo de la papa, que se había extendido a partir de la segunda mitad del siglo XVIII, acusó un gran avance a principios del ochocientos, pero el viñedo se retrajo hasta casi desaparecer a mediados de la centuria tras la crisis del comercio exportador por la pérdida de los mercados exteriores. La cochinilla, que sustituyó a la viña en las zonas costeras, tuvo en el municipio una presencia secundaria (Sánchez-Manzano, 2007, pp. 38-40).

Desde 1820 , la ruina del viñedo junto a la de la barrilla produjo una época de excedente de mano de obra que afectó en especial a los jornaleros, que formaban una clase pobre, de subproletariado, que representó siempre y hasta el siglo pasado una proporción muy alta de la población trabajadora rural, en especial masculina. Los padrones de 1830 sólo registran en los núcleos, pagos y caminos a 74 jornaleros, la mayoría en Los Baldíos y grupos reducidos en San Diego, El Bronco y San Roque. El número de hogares formados por mujeres solitarias o con cabeza de familia femenina, que sobre un total de 453 familias representan en torno a un $10 \%$, no ayuda a explicar la cifra de jornaleros y labradores que se atribuyen a la periferia rural, tanto más porque son aún menos que la de residentes en la ciudad; en sus calles se cuentan 76 jornaleros y 29 labradores, entre los que hay algunas mujeres. Son más acordes con los tiempos las cifras del padrón de 1835, que registra 457 braceros (72\% hombres) y 264 labradores (más del $90 \%$ hombres) en las afueras y pagos de La Laguna (Coderch, 1975 pp. 125126), o el de 1837, para sólo la ciudad, donde braceros y labradores son 175 y 62, respectivamente. El mismo año, en todo el municipio eran 1.213 los activos del primario (859 hombres, el 53,2\% de los activos varones; 354 mujeres, el 26,8 \% de las mujeres activas) también según Coderch. Y en 1860, con una población activa del $41 \%$, el sector primario, integrado en sus dos terceras partes por jornaleros, representaba casi el 70 \% (Quirós, 1971, p. 75).

Una numerosa población trabajadora rural que venía a representar más de cuatro quintas partes del total. En 1791, según el marqués de Villanueva del Prado, no tenía trabajo todo el año y los días en que iba al campo recibía un salario de tres reales de plata y una poca comida; o en especie si era escasa o nula la circulación de dinerario. Ingresos insuficientes para mantener a una familia de cinco miembros, a pesar de no gastar en salud, no sólo por no ser posible pagar al médico sino porque tampoco lo había; ni gastos escolares, porque además de la necesidad del concurso de los niños a la economía familiar, no había escuelas a las que asistir. En general, estos capítulos no aparecen tampoco en los de gastos de las familias campesinas de otras zonas peninsulares. El campesinado fue el sector social que más acusó la crisis económica que se arrastraba desde 1820 (Castro, 1991, p. 158). Y en ese grupo, los jornaleros, que fueron siempre muchos más que los pequeños propietarios, arrendatarios y medianeros, constituyeron la fuerza de trabajo barata, sin yunta ni aperos de labranza, y temporal; esa temporalidad se acompañaba de movilidad y de ahí la presencia en La Laguna, en los meses en que se levantan los dos padrones, de grupos de personas que acuden a la siega «procedentes de las bandas del sur»y que habitan durante ese tiempo en casas de la ciudad. Muy diferente a la estancia temporal de otros transeún- 
tes de Santa Cruz que vienen a la ciudad «por mudanza del temperamento».

Los padrones indican de los labradores si son dueños de yuntas de bueyes y quiénes labran tierras propias o trabajan en ajenas. Ya queda anotado el subregistro en la periferia de la ciudad, donde los padrones sólo registran a 74 jornaleros, 11 labradores y sólo dos oficios más: un carnicero en Viña Nava y un pedrero en San Roque.

De los 29 labradores que residen en la ciudad, 16 se ayudan con yuntas; hay uno con cinco, otro con tres, cuatro con dos, pero uno de ellos, en renta; y seis con una. Además dos labradores tienen venta y uno tiene lonja. Del resto sólo se indica labrador. Trece de ellos tienen domicilio en Herradores, tres en El Tanque, dos en San Juan, y uno en Remojo y Los Molinos. Existe, pues, todavía, un campesino urbano, que tiene la agricultura por ocupación principal, muestra de que La Laguna, como otras muchas ciudades, no es un elemento totalmente diferenciado de sus áreas rurales próximas, con las que mantiene relaciones de reciprocidad e impone su beneficio según la lógica de la jerarquía territorial (García, 1998, p. 141). Por último, de los padrones podemos obtener dos indicadores. Primero: por el reducido número de veces que los propietarios con título aparecen en los padrones, debe deducirse que lo mayor de sus rentas agrarias lo obtienen de otros lugares del municipio, de otras zonas de Tenerife o de otras islas. Segundo: una gran parte de los activos agrícolas trabajan tierras ajenas. De un total de 425 casas en la periferia, el padrón distingue en 309 si están «en lo propio», incluyendo tanto al cabeza de familia como a un familiar, o «en lo de otro»; en el primer caso hay sólo 64 (21\%), en el segundo 217 (70\%), desconociéndose en el resto $(9 \%)$, lo que viene a reflejar la distribución de las tierras, aunque de manera muy elemental.

\section{LOS SIRVIENTES}

La diferenciación de criados y criadas de los oficios y profesiones se apoya en dos circunstancias: su residencia en las casas en las que sirven, lo que aumenta el número de personas por vivienda y reduce el número de hogares con una sola persona; por otra parte, aumentan la población, en especial la femenina, en los tramos de edad en los que se instalan.

En la ciudad hay 958 casas habitadas y de ellas tienen criados 272 y en total hay 487 sirvientes; esto supone que se cuente un criado cada tres casas del total de habitadas y haya un criado cada dos casas de las que tienen alguno. Destacan 22 viviendas que cuentan entre cuatro y ocho
CuAdro II. Número de criados por vivienda

\begin{tabular}{|c|c|c|c|c|}
\hline \multirow{2}{*}{$\begin{array}{c}\text { Criados } \\
\text { por vivienda }\end{array}$} & \multicolumn{2}{|c|}{ Viv. con criados } & \multicolumn{2}{|c|}{ Criados } \\
\hline & núm. & $\%$ & núm. & $\%$ \\
\hline 1 criado & 147 & 53,6 & 147 & 30,2 \\
\hline 2 criados & 74 & 27,1 & 148 & 30,4 \\
\hline 3 criados & 31 & 11,3 & 93 & 19,1 \\
\hline 4 criados & 16 & 5,8 & 64 & 13,1 \\
\hline 5 criados & 3 & 1,1 & 15 & 3,1 \\
\hline 6 criados & 2 & 0,7 & 12 & 2,5 \\
\hline 7 criados & $*$ & $*$ & $*$ & $*$ \\
\hline 8 criados & 1 & 0,4 & 8 & 1,6 \\
\hline TOTAL & 274 & 100,0 & 487 & 100,0 \\
\hline
\end{tabular}

criados; en la del marqués de Vallehermoso, que vive con dos de sus hijos, solteros, y una nieta, se cuentan un mayordomo, un lacayo, un cochero que vive con su mujer y dos hijos, un criado y cuatro criadas, pero hubo un tiempo en que la casa contó con once sirvientes (Pérez, 2009, p. 120); y en la de Pedro Antequera, administrador de Rentas decimales de la provincia, seis criados atienden a los nueve miembros de su familia ${ }^{5}$.

De tres casas con cinco criados, ya vimos que una era la del Obispo, otra la del hacendado Juan Vanderhede. Cuatro tienen 16 casas, entre ellas las del corregidor, la del conde de Siete Fuentes, la de un notario público, la de un coronel retirado, la del capitán y ayudante mayor del Regimiento de Güimar, la de un abogado, la de José González de Mesa, síndico personero..., en el Hospital de Dolores, donde atienden al matrimonio con hija que vive allí, en la fonda de la calle de la Carrera, propiedad de un francés, etc. Es decir, casi todas casas de cargos políticos, militares de rango, profesiones liberales..., que viven en la zona de la ciudad que ocupan las clases altas.

A ello se debe la concentración de sirvientes que se advierte en las calles que se muestra en el Cuadro III. Y porque son, salvo la de la Caza, que es muy corta y en su primer tramo se abre entre un lateral del convento de catalinas y otro de la casa de Alonso de Nava, las más pobladas de la ciudad. En estas calles viven 2.895 personas, que representan más de la mitad de los habitantes urbanos, y ocho de cada diez sirvientes, que son el 13,7 \% de toda la población de la ciudad. Esta concentración es un nuevo elemento para diferenciar este ámbito de La La-

\footnotetext{
${ }^{5}$ Entre ellos a su hijo Juan Bautista Antequera y Bobadilla, nacido en 1823, que hizo la primera circunnavegación a la Tierra en buque blindado.
} 
Cuadro III. Número de criados por viviendas en diez calles centrales de la ciudad

\begin{tabular}{|c|c|c|c|c|c|c|c|c|c|c|c|c|c|}
\hline \multirow{3}{*}{ Calles } & \multicolumn{8}{|c|}{ Núm. de criados por vivienda } & \multirow{3}{*}{$\begin{array}{c}\text { Núm. } \\
\text { de criados }\end{array}$} & \multirow{3}{*}{$\begin{array}{c}\text { Núm } \\
\text { de casas* }\end{array}$} & \multirow{3}{*}{$\begin{array}{c}\text { Núm. } \\
\text { de hab. }\end{array}$} & \multirow{3}{*}{ 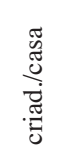 } & \multirow{3}{*}{ 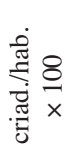 } \\
\hline & 1 & 2 & 3 & 4 & 5 & 6 & 7 & 8 & & & & & \\
\hline & \multicolumn{8}{|c|}{$\mathrm{N}^{\mathrm{o}}$ de viviendas } & & & & & \\
\hline Álamos & 11 & 3 & 2 & 1 & & & & & 27 & 62 & 289 & 0,4 & 9,3 \\
\hline Carrera & 18 & 11 & 6 & 5 & 1 & & & & 83 & 52 & 378 & 1,6 & 21,9 \\
\hline Herradores & 24 & 11 & 5 & 1 & & & & & 65 & 124 & 666 & 0,1 & 9,8 \\
\hline San Agustín & 13 & 11 & 8 & 1 & 1 & 1 & & & 74 & 53 & 336 & 0,7 & 10,4 \\
\hline Moya & 11 & 7 & 3 & 2 & & 1 & & & 48 & 48 & 250 & 1,0 & 9,6 \\
\hline Juan de Vera & 6 & 3 & & 1 & & & & & 16 & 39 & 168 & 0,4 & 9,5 \\
\hline Laurel & 6 & 8 & 1 & & & & & & 25 & 42 & 244 & 0,6 & 10,2 \\
\hline San Juan & 9 & & 2 & & & & & & 15 & 42 & 233 & 0,4 & 6,4 \\
\hline Agua & 2 & 2 & 1 & 1 & 1 & & & 1 & 26 & 35 & 226 & 0,7 & 11,5 \\
\hline Caza & 6 & & 1 & 1 & & & & & 13 & 14 & 89 & 0,9 & 14,6 \\
\hline Pza. Adelantado & & 2 & & & & & & & 4 & 3 & 16 & 1,3 & 25,0 \\
\hline TOTAL & 106 & 58 & 29 & 13 & 3 & 2 & & 1 & 396 & 514 & 2.895 & 0,8 & 13,7 \\
\hline${ }^{*}$ Casas habitadas & & & & & & & & & & & & & \\
\hline
\end{tabular}

guna donde las casas holgadas de las clases altas permiten albergar a un grupo de servidores que, en ocasiones, viven con sus familiares. Se ha señalado ya en la casa de Alonso de Nava y la casuística es muy diversa; hay desde viuda o separada con un hijo, a criada con su madre viuda y dos hijos, en la casa de Vanderhede; o criada casada con marido ausente y dos hijos o criada viuda con dos hijas o criado con su mujer... De esta forma, los índices de ocupación de las casas suben y alcanzan valores de entre el 4,3 habs./casa en Juan de Vera al 6,5 en San Agustín o el 7,3 en la Carrera.

En cuanto a sexo y edad, los 487 sirvientes de la ciudad se reparten en 153 hombres y 334 mujeres; éstas son, pues, el doble que los hombres y suponen el 68,6\% frente al 31,4\% de ellos. El abanico de edades es también más abierto en las criadas que en los criados. El mayor número de sirvientes se agrupa entre los 10 y los 34 años, que en los hombres representan más de las tres cuartas partes y entre las mujeres el $60 \%$; en las edades siguientes, entre los 35 y 59 años, los hombres son ya sólo el $14,4 \%$, mientras las mujeres se mantienen por encima del $30 \%$.

Para el grupo de 10 a 34 años, los criados, que son 119 , alcanzan el $14 \%$ de los hombres de esas edades que viven en la ciudad y las 203 criadas el $17 \%$. En el tramo siguiente, de 35 a 59 años, todavía las criadas son el $12,3 \%$ de las mujeres de esas edades; mientras, los criados han descendido a menos del $5 \%$. A parir de los 25 años se produce un primer descenso y otro mediada la treintena, tanto entre hombres y mujeres. Es, pues, un trabajo para hombres y sobre todo mujeres jóvenes, y urbanos entre los cuales un nuevo trabajo o la emigración y el matrimonio alejan de la servidumbre a una gran parte, mientras un número más pequeño parece acabar fidelizándose al trabajo o a la casa en la que sirven o a ambos.

En el ámbito rural la situación es distinta. El número de criados es muy reducido y si no están afectados de subregistro, aunque esta actividad fue esencialmente urbana, sólo hay 54 y en proporción inversa a la de la ciudad, porque aquí las tres cuartas partes son hombres y sólo una cuarta las mujeres, y el abanico de edades de los hombres se abre hasta los 30-34 años y en las mujeres queda en el tramo 20-24. Aparecen en pequeñas cantidades en el camino de San Miguel, Las Mercedes, Valle Jiménez, La Verdellada, La Cantera y camino de Las Mantecas, que en conjunto suman el $70 \%$ del total. Pero las proporciones de sirvientes respecto al total de personas de su edad se reduce al 8,3\% entre los hombres y el $3,7 \%$ en las mujeres. En estos dos caminos y cuatro pagos se cuentan 38 sirvientes que se reparten en 26 casas, de las cuales 18 tienen un criado y diez un par.

Son muy pocos, en la ciudad y en su periferia, a los que se les anota su procedencia, pero de aquellos de los que se indica muestran un área de reclutamiento amplia 
que incluye lugares a pocos kilómetros, como Guamasa, Tegueste, Tejina o «la montaña», en referencia a los caseríos del cercano macizo de Anaga, y se repiten otros más distantes en el norte de la isla: La Orotava, La Perdoma, El Realejo; o del sur, como Chasna, Arafo o Güimar.

\section{CONCLUSIÓN}

A pesar de las deficiencias advertidas y ya señaladas, los resultados obtenidos del análisis de los padrones parroquiales de La Laguna en 1830 permiten alcanzar algunas conclusiones que muestran algunos rasgos sociales que son propios de la época anterior al periodo constitucional inaugurado con la aprobación de la Constitución en las Cortes de Cádiz. Un número elevado de oficios que a pesar de su diversidad se apoya en los de la construcción y entre los que no aparecen niveles de cierta competencia técnica; y en los del transporte, donde la arriería juega un papel esencial. Notables ausencias entre los artesanos: sastres, relojeros o panaderos, que, si no es por omisión del padrón, se explican mal. Todo ello da idea de una ciudad que, además de verse desposeída de sus antiguas prerrogativas, acusa la inercia y defiende esos antiguos beneficios como si fuesen derechos y en cuyo empeño se encuentran la nobleza y las clases propietarias.

También rasgo de esa pervivencia son los clérigos y los oficios relacionados con la Iglesia, cuyo número supera al de las profesiones liberales, al de cargos y empleos públicos, al de militares, del Ejército y de las milicias, en todos sus rangos, y sólo queda por debajo del comercio y de los oficios. Los agrupados como clero representan el $12 \%$ de los activos que figuran en los padrones. Vive en la ciudad un grupo de labradores que se puede entender como señal de ruralización del espacio urbano; el comercio, apoyado en la «venta» y la lonja, sin apenas presencia de tiendas especializadas, no muestra señales de modernización; el alto número de sirvientes domésticos también es pervivencia del pasado, que aún se mantendrá mucho tiempo. Lo es asimismo el hecho de que las milicias provinciales se mantuviesen después de que fuesen suprimidas en el resto de España. Por último, la localización sobre el plano de las clases altas pone de manifiesto, como en otras ciudades en la misma época, una segregación espacial incompleta que asigna a las clases altas un espacio central exclusivo, pero no excluyente aún, de las clases populares; parte de éstas encuentran albergue en las numerosas infraviviendas, que comparten calle, e incluso portal, con los grupos sociales de rentas altas: viven en «cuartos» en otra época (Pérez, 2009, p. 122), ahora «asesorías», que son estancias de una casa que ocupa una familia distinta y de menor renta que la del titular del inmueble.

\section{BIBLIOGRAFÍA}

Abad Ripoll, E. (2013): «Historia de las Milicias Canarias». Arte y Sociedad, Revista de Investigación, núm. 3, passim

Calero Martín, C. G. (2001): «La Laguna (1800-1936). La modernización de una ciudad en crisis». Revista de Historia Canaria, núm. 183, pp. 51-68.

CAstro Alfin, D. (1991): «Una visión de la agricultura canaria a mediados del siglo XIX», en VIII Coloquio de Historia Canario-Americana. Vol. 2, pp. 151-182.

Cioranescu, A. (1965): La Laguna. Guía histórica y monumental. La Laguna, 292 pp.

- (1977): Historia de Santa Cruz de Tenerife, 4 vols.

Coderch FigueroA, M. (1975): Evolución de la población de La Laguna entre 1750-1860. Instituto de Estudios Canarios, La Laguna, Tenerife, 128 pp.

Hernández Rodríguez, G. (1983): Estadística de las Islas Canarias, 1793-1806, de Francisco Escolar y Serrano. 3 vols., Caja Insular de Ahorros/Centro de Investigación Económica y Social, Las Palmas de Gran Canaria, 436 pp.

JiMÉNEZ DE GREGORIO, F. (1968): «La población de Canarias en la segunda mitad del siglo XVIII». Anuario de Estudios Atlánticos, núm. 14, pp. 127-301.

Moreno Alonso, M. (1980): «Aspectos económicos de Canarias a finales del Antiguo Régimen», en III Coloquio de Historia Canario-Americana. Vol. 1, pp. 294-317.

NúÑEZ Muñoz, M. ${ }^{a}$ F. (1978): «La diócesis de Tenerife. Apuntes para su historia. De los orígenes hasta su restablecimiento definitivo». Revista de Historia de Canarias, XXVI.

Pérez GonzÁlez, R. (1971): «La Laguna». Estudios Geográficos, 124, pp. 443-563.

- (2009): «El centro de San Cristóbal de La Laguna (Tenerife) en 1804. Notas sobre su vecindario», Ería, núm. 78-79, pp. 113-123.

Quintana Andrés, P. C. (2009): «La diócesis de San Cristóbal de La Laguna en los inicios del siglo XIX: el obispo Folgueras Sión, el Cabildo Catedral y la jurisdicción eclesiástica». Boletín Millares Carlo (Centro Asociado unED, Las Palmas de Gran Canaria), pp. 241-269. 
Quirós LinARES, F. (1971): La población de La Laguna (1837-1950). Instituto de Estudios Canarios, La Laguna, $126 \mathrm{pp}$.

- (2004): «Oficios y profesiones en Astorga al final del Antiguo Régimen (el padrón de vecinos de 1836)». Argutorio, núm. 12, pp. 50-53.

SÁnchez-Manzano SuÁrez, F. (2007): La Laguna (18001860). Un estudio de historia agraria. Biblioteca Económica de Canarias.

Santos Puerta, J. (2007): «Situación escolar de Tenerife en 1816-18, de acuerdo con el informe Persiva-Villanueva, realizado por la Real Audiencia en cumpli- miento de Real Orden comunicada por Puig Samper». Historia Educativa (Universidad de Salamanca), núm. 26, pp. 281-298.

Santos Puerto, J., y A. Vega Navarro (2006a): «Estado de la educación en Tenerife a finales del siglo XVIII». Revista de Educación, núm. 339, pp. 661-691.

- y A. Vega Navarro (2006b): «La Comisión de Escuelas de La Laguna y la estadística escolar de Canarias en 1835». Revista de Historia, núm. 188, pp. 221-248.

Tous Meliá, J. (1996): Tenerife a través de la Cartografía (1588-1899). Cabildo de Tenerife, Zona Militar de Canarias, Santa Cruz de Tenerife. 